\title{
WerteWandel
}

Prozesse, Strategien und Konflikte in der gebauten Umwelt 
Kulturelle und technische Werte historischer Bauten

Hg. von Klaus Rheidt und Werner Lorenz

\section{Band 6}


Julia Ess, Eva Maria Froschauer, Elke Richter, Clara Jiva Schulte (Hg.)

\section{WerteWandel}

Prozesse, Strategien und Konflikte in der gebauten Umwelt 
Publiziert mit Unterstützung der Deutschen Forschungsgemeinschaft im Rahmen des Graduiertenkollegs 1913 »Kulturelle und technische Werte historischer Bauten«, Brandenburgische Technische Universität Cottbus-Senftenberg; Leibniz-Institut für Raumbezogene Sozialforschung Erkner; Archäologisches Institut der Humboldt Universität zu Berlin.

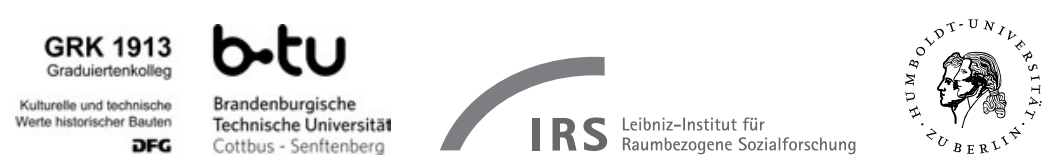

Wissenschaftlicher Beirat

Prof. Dr. phil. Christoph Bernhardt, Prof. Dr. phil. Burcu Doğramacı, Dr. phil. Dipl.-Ing. Martin Düchs, Dr.-Ing. Sabine Hansmann, Dr. phil. Nura Ibold, Prof. Dr.-Ing. habil. Hans-Georg Lippert, Dr. phil. Christine Neubert, Dr.-Ing. Katja Piesker, Dr. phil. Britta Rudolff, Dr.-Ing. Joseph Rustom, Prof. Dr. phil. Christiane Salge, Dr. phil. Achim Saupe, Prof. John Schofield PhD, Dr. rer. nat. Annette Voigt, Prof. Dipl.-Ing. M.Arch. (Cornell) Thomas Will, Albrecht Wiesener M.A.

Konzept: Julia Ess, Eva Maria Froschauer, Elke Richter, Clara Jiva Schulte

Projektkoordination: Albrecht Wiesener, Sophia Hörmannsdorfer

Lektorat: Johannes Althoff, William Hatherell

Layout, Satz und Redaktion: Sophia Hörmannsdorfer

Covergestaltung: Jörg Denkinger

Druck und Bindung: Beltz Grafische Betriebe $\mathrm{GmbH}$, Bad Langensalza

Umschlagabbildung: Berlin-Marzahn, Dorf, Neubaugebiet, BArch, Bild 183-1985-0723-003 (Foto: Zimmermann).

Library of Congress Control Number: 2021935904

Bibliografische Information der Deutschen Nationalbibliothek

Die Deutsche Nationalbibliothek verzeichnet diese Publikation in der Deutschen Nationalbibliografie; detaillierte bibliografische Daten sind im Internet über http://dnb.dnb.de abrufbar.

Dieses Werk ist urheberrechtlich geschützt. Die dadurch begründeten Rechte, insbesondere die der Übersetzung, des Nachdrucks, des Vortrags, der Entnahme von Abbildungen und Tabellen, der Funksendung, der Mikroverfilmung oder der Vervielfältigung auf anderen Wegen und der Speicherung in Datenverarbeitungsanlagen, bleiben, auch bei nur auszugsweiser Verwertung, vorbehalten. Eine Vervielfältigung dieses Werkes oder von Teilen dieses Werkes ist auch im Einzelfall nur in den Grenzen der gesetzlichen Bestimmungen des Urheberrechtsgesetzes in der jeweils geltenden Fassung zulässig. Sie ist grundsätzlich vergütungspflichtig. Zuwiderhandlungen unterliegen den Strafbestimmungen des Urheberrechts.

Dieses Buch ist auch als E-Book (ISBN PDF 978-3-0356-2327-7) erschienen.

ISBN 978-3-0356-2324-6

(C) 2021 Birkhäuser Verlag GmbH, Basel

Postfach 44, 4009 Basel, Schweiz

Ein Unternehmen der Walter de Gruyter GmbH, Berlin/Boston 